\title{
Comparative Analysis of CT and MRI in Emergency Assessment of Stroke: A Review
}

\author{
Digvijay Singh and Chanchal Kaushik ${ }^{*}$ \\ Chitkara School of Health Sciences, Chitkara University, Punjab-140401, India \\ "Email: chanchal.kaushik@chitkara.edu.in
}

\section{ARTICLE INFORMATION}

Received: November 30, 2018

Revised: February 04, 2019

Accepted: March 08, 2019

Published Online: April 08, 2019

\section{Keywords:}

Stroke, Ischemic stroke, Hemorrhagic stroke, Infarcts, Cerebrovascular accidents

\section{ABSTRACT}

Objective: To compare the diagnostic accuracy of computed tomography (CT) and magnetic resonance imaging (MRI)in emergency assessment of stroke in brain imaging from the review of literature.

Method: Relevant databases (PubMed, google scholar etc.) were searched and literature were reviewed from 1995 to 2019. Literature from non-Scopus and unauthorized authorizations was excluded.

Result: It was observed that for MRI DWI (Diffusion-weighted imaging) is preferred and in CT, axial sections are opted. In earlier studies, it was seen that neither CT nor MRI came out to besuperior. This may be due to the previous technology used. Some studies also, suggested that Diffusion-weighted imaging is highly accurate in diagnosis of stroke and also superior to CT. Another study suggested that SWI is a new approach in visualizing the hemorrhage in acute stroke. On one hand, evidence revealed that MRI is as good as CT. While on the other hand, literature concluded that CT angiography is good for intracranial and extracranial vasculature. Some studies suggested that $\mathrm{CT}$ is more reliable and is readily available for stroke.

Conclusion: Present study concludes that both diagnostic imaging modalities i.e., CT and MRI have their advantages in diagnosis of ischemic and hemorrhagic stroke.Also chances of stroke increases with increase in age. Other factors influencing the stroke diagnosis and treatment are type of stroke, diagnostic imaging modality available, and cost-effectiveness of diagnostic exams performed.

\section{Introduction}

Stroke is a medical condition which arises when blood flow to an area of the brain is cut off or stops. It has been seen that stroke is the most persistent and leading cause of death after coronary artery disorder (GBD 2015 Disease and Injury Incidence and Prevalence Collaborators, 2016). It is categorized according to its causes such as Ischemic stroke (caused by the insufficient blood supply to the brain) and Hemorrhagic stroke (caused by bursting of blood vessels in the brain) and TIA (Transient Ischemic Stroke) i.e. short term stroke can be treated within 6 hours from the beginning of symptoms (stroke). There are several factors responsible for stroke but the main risk factorsare high blood pressure; excessive weight, physical inactivity, atherosclerosis, smoking, obesity, high blood cholesterol, and diabetes (Risk factor for stroke). Stroke generally occurs in the age group of 50-55, but due to poor and unhealthy lifestyle and leading risk factors, it can happen at any age. It can be diagnosed through severaldiagnostic techniques like a blood test, electrocardiogram (ECG), carotid ultrasound, cerebral angiogram, computed tomography (CT) and magnetic resonance imaging (MRI). CT scan helps in detecting and diagnosing different densities and tissue, provides the detailed information about organs and their vessels. Advancement in CT scanner helps in patient comfort, reduced scanning time and high resolution images.

Blood vessels supply oxygen and nutrients to the brain. Ischemic stroke results due to blockage in an artery. Narrowing or blockage of the artery of the neck and brain is seen as the most common cause. It is often caused by the deposition of cholesterol or plaque in the vessels, resulting in 
blocking the supply of oxygen to several parts of brain. This Ischemic stroke is of two type's i.e. thrombotic and embolic stroke. In a thrombotic stroke, the artery or cerebral artery is blocked by the formation of a clot in the brain itself. While in the embolic stroke, a clot formation occurs in an area other than brain.In hemorrhagic stroke, results due to the rupturing of blood vessels of the brain. There are two types of hemorrhagic stroke i.e. intracerebral or subarachnoid. In an intracerebral hemorrhage, rupturing or bleeding of arteries happens within the brain itself. It is also known as cerebral bleed or intracranial hemorrhage. It can take place within the tissue or the ventricles. Its symptoms include headache, weakness, vomiting, seizures, stiffness of the neck, etc. The main causes are brain trauma, aneurysm, tumors, etc. and the risk factors are hypertension, alcohol, and drug usage (Caceres et al., 2012). Subarachnoid Hemorrhage (SAH) is rupturing or bleeding of the vessels outside the brain tissue but within the skull i.e. between arachnoid and pia mater (Abraham et al., 2015). Its symptoms are almost same as of intracranial hemorrhage like headache, vomiting, fever, seizures, etc. Risk factors includes hypertension, smoking, family history, alcohol, and drug use, etc. It is usually detected by CT scan within 6 hours from the onset. Epidural hemorrhage (Hematoma) includes bleeding or rupturing of the vessel between the skull and dura mater. Symptoms may include loss of consciousness, vomiting, headache, confusion, trauma, etc. Subdural hemorrhage (SDH) is the bleeding of the vessel within subdural space. It leads to an increase in the compulsion inside the skull, which may result in damage to the delicate brain. Symptoms include loss of consciousness, pain, headache, dizziness, vomiting, inability to speak, etc.

$\mathrm{CT}$ is a diagnostic imaging technique which utilizes $\mathrm{X}$-rays for multiple projections and forms sections or slices of area of interest. Digital processors allows for generating three-dimensional images. CT scan of the head is done to determine infarcts, cancers, hemorrhage, calcifications of brain tissue, and injury to the head. The dark area i.e. hypo densities shows edema and infarcts. The bright area i.e. hyper densities shows calcifications and acute hemorrhages and injuries to the brain are visualized with the help of bone windows. Clinical indications for CT head are many including head trauma, stroke, headaches, seizures, hematoma, hydrocephalus, bleeding, brain infection, tumors, and birth defects. A CT head can also be done for behavioral changes, fainting, muscle weakness, loss of vision, numbness, loss of hearing, tingling sensation, etc. MRI or NMRI (Nuclear magnetic resonance imaging) is an imaging technique which uses a strong magnetic field, radio frequency signals and gradient coils to produce the image of body tissues. Clinical indications for MRI brain includes birth defect, bleeding, multiple sclerosis, tumors etc. An
MRI head can also be done for behavioral changes, fainting, muscle weakness, loss of vision, numbness, loss of hearing, tingling sensation, etc. Unlike, CT scan which utilizes $\mathrm{X}$-rays, which is an ionizing radiation, $\mathrm{MRI}$ is a non-ionizing modality and utilizes strong magnetic field, gradients coil, RF signals to produce images of body. The contrast in CT scan is based on the attenuation of X-rays in patient's body, while different properties of soft tissue are used to enhance the contrast in MRI images. By variation in parameters of the scans, tissue contrast can be modified to enhance the images. Both CT and MRI images can be enhanced by the use of dye known as contrast agents. In CT iodinated contrast agents used such as iohexol, Visipaque etc. While in MRI contrast agents with paramagnetic properties are used such as gadolinium based contrast media. The MRI contrast agents are contraindicated (a particular condition in which drug should not be used) inhigh risk patients. CT scan gives more detailed information on head injuries, stroke occurrence, brain tumors, and other diseases of the brain. On the other hand, MRI provides detailed soft-tissue information than CT. Both the diagnostic modalities have their advantages and disadvantages for a particular scan. Recent advances in diagnostic imaging and radiology have led to better diagnostic information obtained via these scans and also provides minute details of the organs or tissues being scanned. CT scan is always used as a primary method for distinguishing the stroke and its types. CT produces images in less time and MRI uses DWI which provides detailed information on stroke.

Disagreements have been seen in deciding which diagnostic technique is superior and should be preferred during the emergency assessment of stroke in patients. CT imaging is comparatively less expensive and consumes less time in scanning as compared to the MRI. The early diagnosis and treatment of stroke is of outmost importance and it is also essential to know the type of stroke before its treatment. Early diagnosis of stroke or predictingits occurrence is crucial. Researchers are actively involved in studies which utilizes brain imaging techniques to identify high risk patient's stroke. This study aims to evaluate the recent trends in diagnosis and detection of ischemic and hemorrhagic stroke from existing literature review.

\section{Literature Review and Discussion}

The published literature from 1995 to 2019 (extracted from google scholar, PubMed etc.) was reviewed in depth and a comparative analysis was drawn. Literature from nonScopus and unauthorized sources was excluded. Results of Urbach et al. in 2000 showed that the diffusion-weighted pulse sequence used in MRI has high detection and diagnosis rate when it is compared to a normal CT scan. 



Figure 1: The left side shows that there is no confirmation of acute infarct in the NCCT head, Right shows the infarction of full left side middle cerebral artery territory on the same patient after 20 minutes in MRI scan.

There is a high occurrence of susceptibility and carefulness of DWI-MR on scanning for outpatients with ischemic infarcts in some patients. The authors concluded that a factor of higher sensitivity during acute cerebral infarcts could help in choosing an ideal imaging modality during a stroke (Urbach et al., 2000). Oppenheim et al. in 2000 also reported that MRI has the highest sensitivity as well as specificity for the diagnosis of acute ischemic stroke. The authors reported that MRI is used to visualize the shape, size, and size of the lesions also provides detailed and minute information on stroke. They concluded that MRI can be used as a routine imaging modality for stroke cases and also in emergency setups. The use of MRI as an emergency tool for the detection of stroke depends greatly on its availability (Oppenheim et al., 2000). Lansberg et al. in 2000 compared the sensitivity, specificity, and reliability of Diffusion-weighted MRI with CT to determine the acute intracerebral infarcts. The authors reported a higher distinguishing exactness of DWI- MRI for cerebral infarcts than compared to CT scans. CT is not a much sensitive and reliable tool for lesion detections. It also concluded that further studies may be needed to detect high accuracy of DW MRI for the detection of stroke. MRI provides minute details of the lesions and more accurately detects infarctions (Lansberg et al., 2000). Fiebach et al. in 2001 reported that images shown in MRI are much more reliable and accurate for acute ischemic lesions as compared to CT which is not much reliable. CT is mostly used for stroke diagnosis due to its fast scanning time but DWI gives more defining lesions for detection and reliability of acute stroke. This is the area of concern that whether CT or MRI produces more accurate information and is used in emergency assessment of stroke patients (Fiebach et al., 2001). Fiebach et al. in 2002 in another study said that DWI MRI is always a preferred choice for the detection of acute infarcts. The authors reported that there is a significant difference in the results of both CT imaging and MRI imaging after the beginning of indications of stroke. Earlier CT is performed first followed by MRI to give progressive results, still, DW MRI gives a more detailed information of it. The diffusion-weighted sequence is still under research for further development (Fiebach et al., 2002). MD et al. in 2004 compared Susceptibility weighted images (SWI) with standard MRI scan and NCCT brain scans. The authors presented the new SWI sequence is much thoughtful and is a new approach for the detection of acute stroke as compared to CT and conventional MRI. SWI is sufficient for the detection of acute hemorrhage and could eliminate the need for CT, but there is further need for investigation on these sequences (Wycliffe et al., 2004). Wardlaw et al. in 2003 in their study reported that CT scanning is not able to recognize hemorrhages, and in order to distinguish the types of hemorrhages it should be done within few days. For patientswith late clinical indications, MRI scan may be preferred (Wardlaw et al., 2003). Kidwell et al. in 2004 reported that CT and MRI both imaging modalities are accurate, sensitive, and reliable in the detection of acute hemorrhage with stroke symptoms. Both imaging modalities provide equal results for patients with hemorrhagic stroke. Also for chronic hemorrhagic stroke, MRI is a good choice of modality as compared to CT. CT is as sensitive as MRI in the detection of acute hemorrhagic stroke (Kidwell et al., 2004). Bisdas et al. in 2004 reported that both MRI and CT provides equal results for the assessment of patients with stroke. There is no significant difference between these imaging modalities (Bisdas et al., 2004). In a literature review of 2004, Desal et al. studied the need for emergency assessment of all cerebrovascular accidents i.e. stroke, to know its type and better visualization of disease. The authors said that the advancement in neurology imaging has led to better assessment and care of all strokes and types. Earlier CT was a choice of modality to discriminate the hemorrhage and ischemia, but now MRI is used for better and initial assessment of stroke patients. It can discriminate between acute stroke, sub-acute stroke, and chronic stroke. It is understood from literature that DWI in MRI have high sensitivity and specificity for acute infarcts and different lesions. It is concluded that MRI is highly effective in the determination and diagnosis of acute stroke and is a choice of modality for the initial assessment of stroke (Desal et al., 2004). According to Barber et al. (2005), there exists a disagreementfor selecting the optimal imaging modality for the diagnosis of stroke. The authors concluded that an optimal imaging modality for initial and emergency assessment of acute stroke and infarcts should depend on its feasibility and its availability. CT scan is fast and mostly available in all clinical setups therefore it is used for initial 
assessment of stroke patients, also there exists no significant difference in the results of $\mathrm{CT}$ and MRI.

Hence, it is indicated that better imaging modality for neuroimaging depends upon its versatility and availability (Barber et al., 2005). Kidwell et al. 2006 presented the advantages and disadvantages of $\mathrm{CT}$ and MRI in neuroimaging for assessment of acute stroke. It also resonates with the work of Kidwell et al. 2004 that both the imaging modalities play a key part in the control of cerebrovascular disease particularly infarcts. Neurology imaging research gives pivotal details of the trauma of tissue i.e. shape, size, area, the occurrence of ischemic and hemorrhagic stroke as well as its reoccurrence, the position of the vessel (area and strength of stenosis and obstructions), and cerebral expulsion (size, site, and strength) (Kidwell et al., 2006). Chalela et al. in 2007, prospectively compared both imaging modalities and reported that neither CT nor MRI is $100 \%$ sensitive for the detection of stroke, although MRI is more accurate and reliable in the detection of both hemorrhagic and ischemic stroke. It can detect both acute and chronic hemorrhage and therefore is the preferred test for early detection of stroke. Thus we can conclude that in most of the literature studied so far MRI is a better imaging modality for initial assessment of stroke (Chalela et al., 2007). Wintermark et al. 2007 reported that the association of MRI and CT angiography is magnificent for shape, size, and theimplication of infarcts, for obstruction of cerebral arteries, umbra, or infarct proportion (Wintermark et al., 2007).

Depending on MRI or CT angiography both show the same diagnostic conclusion in all types of stroke. Khandelwal in 2008, reported that if the infarcts is not diagnosed earlier it can cause muscle weakness, vision or speech loss, difficulties in carrying day to day activity, or eventually death. Khandelwal shows that both CT perfusion and MR perfusion can be used for diagnosis of stroke but still MRI perfusion has a more accurate and reliable technique for its assessment (Khandelwal, 2008). There is another study in which Hacke et al. 2009 reported that the DIAS study did not display a profit of desmoteplase given three to nine hours following the arrival of stroke. The elevated response rate in the group could be expressed by mild stroke data (low baseline NHAS score, small core lesion and small mismatch volumes that were organized with no vessel obstructions) which possibly deducts the possibility to detect any impact (Hacke et al., 2009). Schellinger et al., 2010 said that DWI is convenient and must be practiced further than NCCT for identification of ischemic infarct between twelve hours of clinical indications. DWI helps in precise identification of ischemia. Although the delicacy of DW for the identification of ischemic stroke in a common specimen of the victim with severe infarcts is not ideal (Schellinger et al., 2010). An Indian review of literature by Narayan et al., 2010 which shows the probability profiles, hazards, gender-related to the chance of acute cerebral venous thrombosis. The authors suggest that the highest prevalence of the disease has mostly occurred in males than in females. There are several risk factors, most commonly anemia, drug use, alcohol, and poor lifestyle resulting in increased occurrence of stroke (Narayan et al., 2010). Brazzelli et al., 2010 prospectively compared the CT and MRI for the detection of hemorrhagic and ischemic stroke. It is concluded by the authors that DWI in MRI is much sensitive than CT, but not significantly specific in the detection of acute hemorrhage. There are limited studies in this area and with different methodological approaches applied (Brazzelli et al., 2010). According to Lovblad et al, 2010 both diagnostic and therapeutic techniques are under research. The authors suggest that due to advancements in medical imaging techniques, CT is the most reliable and a faster approach for the detection of acute ischemic hemorrhage. This resonates with some other studies about CT being a faster and reliable tool (Lovblad et al., 2010). Coutts et al. 2012 reported that early assessment of the intracranial and extracranial vascular lesions using CT or CT angiography could anticipate the recurrence of stroke and indications in outpatients with TIA and minimal stroke.

In many centers, CT or CTA is mostly available than MRI scan, and physicians could prefer to the modality which is easily available in a particular center (Coutts et al., 2012). Biesbroek et al., 2013 reported that CT perfusion is an essential tool in the detection of infarcts. CT perfusion is readily available in many institutions. A systematic review presented high sensitivity and specificity of CT perfusion in the diagnosis of most infarcts (Biesbroek et al., 2013). Ferguson et al., 2018 said that the standardized visual rating scales of white matter lesions and atrophy mostly presents substantial agreement between CT and MRI. Clinical CT scans have a strong potential for wider exploitation in research studies, particularly in intensely unwell population (Ferguson et al., 2018). Another study of Chen et al., in 2019 with a tissue-specific threshold for grey matter and white matter presents a more accurate estimation of acute ischemia (Chen et al., 2019). Mejdoubi et al., 2010 said that CTP or CT perfusion is used as a routine investigation for determination and characterization of stroke and also can be used when MRI is not available in the imaging center (Mejdoubi et al., 2010).

\section{Conclusion}

Neurology imaging plays a pivotal role in the assessment of acute stroke. While the disease is diagnosed through patient's symptoms and clinical examinations, brain imaging can authenticate the presence of disease and differentiate between the stroke and its type's i.e. hemorrhagic and 
ischemic stroke. The prompt and correct detection of infarcts and their types is crucial for decision making in the treatment delivery. The ideal imaging modality should provide emergency assessment of stroke, correct detection of both ischemic and hemorrhagic stroke and differentiation of CVA causes with others (Mejdoubi et al., 2010). The literature studied so far concludes that DWI in MRI is accurate and highly sensitive and specific in comparison to CT scan. CT is suggested as a faster and reliable tool for assessing infarcts by some studies. Also, predominance of strokeis mostly seen in males than in females. CT and MRI have their advantages in case of diagnosis of ischemic and hemorrhagic stroke. There are limited studies on contrast enhanced imaging to conclude for image quality in ischemic and hemorrhagic stroke.Also, further studies are needed to conclude if MRI can be an optimal choice of modality to diagnose acute stroke in routine clinical practices. Also, further studies should be conducted representing sample population closely and suggesting in decision making for detection of acute stroke incorporating cost effectiveness and other parameters as well.

\section{References}

Abraham, M.K., \& Chang, W.T.W. (2016). Subarachnoid hemorrhage. Emergency Medicine Clinics, 34(4), 901916. https://doi.org/10.1016/j.emc.2016.06.011.

Albers, G.W., et al. (2018). Thrombectomy for stroke at 6 to 16 hours with selection by perfusion imaging. The New England Journal of Medicine, 378(8), 708-718. https://doi.org/10.1056/NEJMoa1713973

Barber, P.A., Hill, M.D., Eliasziw, M., Demchuk, A. M., Pexman, J.H.W., Hudon, M. E., Tomanek, A., Frayne, R. \& Buchan, A.M. (2005). Imaging of the brain in acute ischaemic stroke: comparison of computed tomography and magnetic resonance diffusionweighted imaging. Journal of Neurology, Neurosurgery \& Psychiatry, 76(11), 1528-1533.

https://doi.org/10.1136/jnnp.2004.059261

Biesbroek, J.M., Niesten, J.M., Dankbaar, J.W., Biessels, G.J., Velthuis, B.K., Reitsma, J.B., \& Van Der Schaaf, I.C. (2013). Diagnostic accuracy of CT perfusion imaging for detecting acute ischemic stroke: a systematic review and meta-analysis. Cerebrovascular diseases, 35(6), 493501. https://doi.org/10.1159/000350200

Bisdas, S., Donnerstag, F., Ahl, B., Bohrer, I., Weissenborn, K., \& Becker, H. (2004). Comparison of perfusion computed tomography with diffusion-weighted magnetic resonance imaging in hyperacute ischemic stroke. Journal of computer assisted tomography, 28(6), 747-755.

https://doi.org/10.1097/00004728-200411000-00004
Brazzelli, M., Sandercock, P.A.G., Celani, M.G., Righetti, E., Chappell, F.M., Arestis, N., Wardlaw, J.M. \& Deeks, J. J. (2010). MRI versus CT for detection of acute vascular lesions in patients presenting with stroke symptoms. Stroke, 41(5), e427-e428. https://doi.org/10.1161/STROKEAHA.109.568667

Caceres, J.A., \& Goldstein, J.N. (2012). Intracranial hemorrhage. Emergency medicine clinics of North America, 30(3), 771. https://doi.org/10.1016/j.emc.2012.06.003

Carpenter, C.R., Hussain, A.M., Ward, M.J. Zipfel, G.J., Fowler, S., Pines, J.M. \& Sivilotti, M.L.A. (2016). Spontaneous Subarachnoid Hemorrhage: A Systematic Review and Meta-analysis Describing the Diagnostic Accuracy of History, Physical Examination, Imaging, and Lumbar Puncture with an Exploration of Test Thresholds. Academic Emergency Medicine. 23(9), 963-1003. https://doi.org/10.1111/acem.12984

Chalela, J.A., Kidwell, C.S., Nentwich, L.M., Luby, M., Butman, J. A., Demchuk, A. M., Hill, M.D., Patronas, N., Latour, L. \& Warach, S. (2007). Magnetic resonance imaging and computed tomography in emergency assessment of patients with suspected acute stroke: a prospective comparison. The Lancet, 369(9558), 293-298. https://doi.org/10.1016/S0140-6736(07)60151-2

Chen, C., Bivard, A., Lin, L., Levi, C. R., Spratt, N. J., \& Parsons, M. W. (2019). Thresholds for infarction vary between gray matter and white matter in acute ischemic stroke: a CT perfusion study. Journal of Cerebral Blood Flow \& Metabolism, 39(3), 536-546. https://doi.org/10.1177/0271678X17744453

Coutts, S.B., Modi, J., Patel, S.K., Demchuk, A.M., Goyal, M., \& Hill, M. D. (2012). CT/CT angiography and MRI findings predict recurrent stroke after transient ischemic attack and minor stroke: results of the prospective CATCH study. Stroke, 43(4), 1013-1017. https://doi.org/10.1161/STROKEAHA.111.637421

Desal, H.A., Auffray-Calvier, E., Guillon, B., Toulgoat, F., Madoz, A., Kersaint-Gilly, A.D. \& Pasco-Papon, A. (2004). Emergency imaging of cerebrovascular accidents. Journal of Neuroradiology, 31(4), 327-333. https://doi.org/10.1016/S0150-9861(04)97011-3

Fiebach, J., Jansen, O., Schellinger, P., Knauth, M., Hartmann, M., Heiland, S., Ryssel, H., Pohlers, O., Hacke, W. \& Sartor, K. (2001). Comparison of CT with diffusion-weighted MRI in patients with hyperacute stroke. Neuroradiology, 43(8), 628-632 https://doi.org/10.1007/s002340100542

Fiebach, J.B., Schellinger, P.D., Jansen, O., Meyer, M., Wilde, P., Bender, J., Schramm, P., Juttler, E., Oehler, 
J., Hartmann, M., Hahnel, S., Knauth, M., Hacke, W., \& Sartor, K. (2002). CT and diffusion-weighted MR imaging in randomized order: diffusion-weighted imaging results in higher accuracy and lower interrater variability in the diagnosis of hyperacute ischemic stroke. Stroke, 33(9), 2206-2210.

https://doi.org/10.1161/01.STR.0000026864.20339.CB

Ferguson, K.J., Cvoro, V., MacLullich, A.M.J., Shenkin, S. D., Sandercock, P.A.G., Sakka, E., \& Wardlaw, J.M. (2018). Visual rating scales of white matter hyperintensities and atrophy: comparison of computed tomography and magnetic resonance imaging. Journal of Stroke and Cerebrovascular Diseases, 27(7), 1815-1821. https://doi.org/10.1016/j. jstrokecerebrovasdis. 2018.02.028

GBD 2015 Disease and Injury Incidence and Prevalence Collaborators (2016). Global, regional, and national incidence, prevalence, and years lived with disability for 310 diseases and injuries, 1990-2015: a systematic analysis for the Global Burden of Disease Study 2015. The lancet, 388(10053), 1545-1602. https://doi.org/10.1016/S0140-6736(16)31678-6

Hacke, W., et al. (2009). Intravenous desmoteplase in patients with acute ischaemic stroke selected by MRI perfusion-diffusion weighted imaging or perfusion CT (DIAS-2): a prospective, randomised, double-blind, placebo-controlled study. The Lancet Neurology, 8(2), 141-150.

https://doi.org/10.1016/S1474-4422(08)70267-9

https://en.wikipedia.org/wiki/CT_scan

https://en.wikipedia.org/wiki/Magnetic_resonance_ imaging

Khandelwal, N.(2008).CT perfusion in acute stroke. Indian journal of radiology and imaging, 18(4), 281-286. https://doi.org/10.4103/0971-3026.43837

Kidwell, C.S., et al. (2004). Comparison of MRI and CT for detection of acute intracerebral hemorrhage. Jama, 292(15), 1823-1830.

https://doi.org/10.1001/jama.292.15.1823

Kidwell, C. S., \& Hsia, A. W. (2006). Imaging of the brain and cerebral vasculature in patients with suspected stroke: advantages and disadvantages of CT and MRI. Current neurology and neuroscience reports, 6(1), 9-16. https://doi.org/10.1007/s11910-996-0003-1

Lansberg, M.G., Albers, G.W., Beaulieu, C. \& Marks, M.P. (2000). Comparison of diffusion-weighted MRI and CT in acute stroke. Neurology, 54(8), 1557-1561. https://doi.org/10.1212/WNL.54.8.1557

Lövblad, K.O., \& Baird, A.E. (2010). Computed tomography in acute ischemic stroke. Neuroradiology, 52(3), 175187. https://doi.org/10.1007/s00234-009-0633-5
Narayan, D., Kaul, S., Ravishankar, K., Suryaprabha, T., Bandaru, V.C.S.S., Mridula, K.R., Jabeen, S.A., Alladi, S., Meena, A.K. \& Borgohain, R. (2012). Risk factors, clinical profile, and long-term outcome of 428 patients of cerebral sinus venous thrombosis: Insights from Nizam's Institute Venous Stroke Registry, Hyderabad (India). Neurology India, 60(2), 154. https://doi.org/10.4103/0028-3886.96388

Oppenheim, C., Logak, M., Dormont, D., Lehericy, S., Manai, R., Samson, Y., Marsault, C. \& Rancurel, G. (2000). Diagnosis of acute ischaemic stroke with fluid-attenuated inversion recovery and diffusionweighted sequences. Neuroradiology, 42(8), 602-607. https://doi.org/10.1007/s002340000356

Schellinger, P.D., Bryan, R.N., Caplan, L.R., Detre, J.A., Edelman, R.R., Jaigobin, C., Kidwell, C.S., Mohr, J.P., Solan, M., Sorensen, A.G. \& Warach, S. (2010). Evidence-based guideline: the role of diffusion and perfusion MRI for the diagnosis of acute ischemic stroke: report of the Therapeutics and Technology Assessment Subcommittee of the American Academy of Neurology. Neurology, 75(2), 177-185. https://doi.org/10.1212/WNL.0b013e3181e7c9dd

Urbach, H., Flacke, S., Keller, E., Textor, J., Berlis, A., Hartmann, A., Reul, J., Solymosi \& Schild, H. H. (2000). Detectability and detection rate of acute cerebral hemisphere infarcts on CT and diffusionweighted MRI. Neuroradiology, 42(10), 722-727. https://doi.org/10.1007/s002340000401

Wardlaw, J.M., Keir, S.L., \& Dennis, M.S. (2003). The impact of delays in computed tomography of the brain on the accuracy of diagnosis and subsequent management in patients with minor stroke. Journal of Neurology, Neurosurgery \& Psychiatry, 74(1), 77-81. https://doi.org/10.1136/jnnp.74.1.77

Wintermark, M., Meuli, R., Browaeys, P., Reichhart, M., Bogousslavsky, J., Schnyder, P. \& Michel, P. (2007). Comparison of CT perfusion and angiography and MRI in selecting stroke patients for acute treatment. Neurology, 68(9), 694-697.

https://doi.org/10.1212/01.wnl.0000255959.30107.08

Wycliffe, N.D., Choe, J., Holshouser, B., Oyoyo, U.E., Haacke, E.M., \& Kido, D.K. (2004). Reliability in detection of hemorrhage in acute stroke by a new three-dimensional gradient recalled echo susceptibility-weighted imaging technique compared to computed tomography: a retrospective study. Journal of Magnetic Resonance Imaging, 20(3), 372377. https://doi.org/10.1002/jmri.20130 


\section{旬 \\ CHITKARA}

\section{Journal of Multidisciplinary Research in Healthcare}

Chitkara University, Saraswati Kendra, SCO 160-161, Sector 9-C, Chandigarh, 160009, India

Volume 5, Issue 2

April 2019

ISSN 2393-8536

Copyright: [C 2019 Digvijay Singh and Chanchal Kaushik] This is an Open Access article published in Journal of Multidisciplinary Research in Healthcare (J. Multidiscip Res. Healthcare) by Chitkara University Publications. It is published with a Creative Commons Attribution- CC-BY 4.0 International License. This license permits unrestricted use, distribution, and reproduction in any medium, provided the original author and source are credited. 